\title{
Research on the application of a new platform of the robot as an active support
}

\author{
KorganbaySholanov ${ }^{1, ~ a ~}$,Utebayev Ruslan ${ }^{2, b}$ \\ ${ }^{1}$ Kazakh National Science Technical University, Republic of Kazakhstan,Almaty \\ ${ }^{2}$ Kazakh National Science Technical University, Republic of Kazakhstan,Almaty \\ ashoklor@rambler.ru*, butebaev_r@mail.ru
}

Keywords:Platform type robot, functionality, motor reaction, controllable hydraulic drive, active support.

Abstract.The paper describes a new six mobility platform type robot SHOLKOR. Due to its unique properties, the new platform type robot can be used as an active support to protect buildings, facilities and machinery from sudden mechanical impacts.

This paper includes a description of the experimental assembly comprising a platform type robot with automatically controlled hydraulic drives. To assess the possibility to use the robot as an active support, it is proposed to consider the motor reaction and to determine the motor reactions when designing the drives through computer generated simulation using Matlab Simulink. To analyse the motor reactions of actual single-side drivers with a pull-back spring with throttling channels in the piston and without channels, experimental research was conducted with the experimental assembly. The experimental findings allowed for developing recommendations for the use of the new platform type robot as an active support.

\section{Introduction}

Several platform type robots are currently used in various fields. Such robots have structure topology similar to the Gough-Stewart platform [1-3]. The advantages of these robots are generally known. However, in the majority of platform type robots with more than 3 degrees of freedom, the variable coordinates of the movable platform are interrelated with each other so that a movement of any drive requires simultaneous coordinated movement in the other drives connecting the platforms. There is no apparent solution for the direct kinematic problem for parallel manipulators of such platform type robots [3-6].Therefore, certain issues are encountered when creating control systems, as well as during their operation, because failure in one drive results in inoperability of the entire robot. To improve functionality of the parallel manipulators of a platform type robot, a new structure topology of the parallel manipulator was offered. This structure topology has ensured a number of benefits compared to the existing Gough-Stewart schemes.

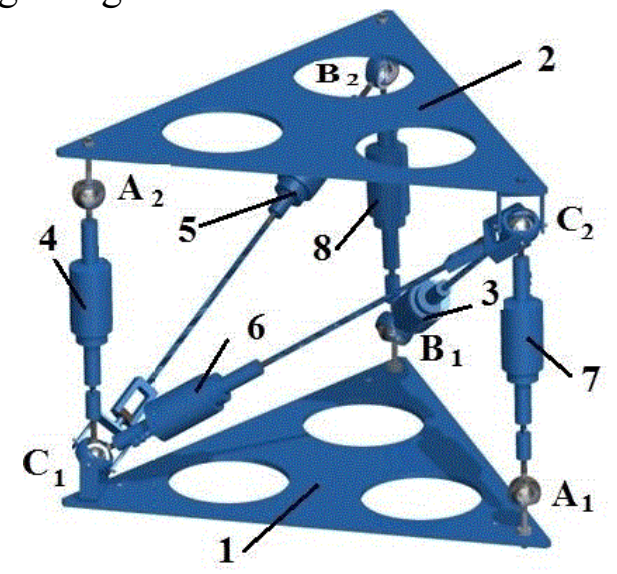

Fig. 1 Model of the parallel manipulator

A new platform type robot based on a new structure topology was offered in work $[7,8]$. The robot consists of two platforms connected with six connecting links (CL), which allow linear movements. The model of the parallel manipulator is shown in the structure scheme in Fig.1, where: 1 
- fixed platform, with regards to which movable platform 2 makes its movements. Fig. 1 also shows connecting links 3-8 with drives, which connect the lower platform 1 and upper platform 2 with multilink spherical joints. A CL forms with the platforms in the following units: $A_{1}, A_{2}-$ two link spherical joints; $B_{1}, B_{2}-$ three link spherical joints; $C_{1}, C_{2}-$ four link spherical joints.Based on the model (Fig.1), a working model of the parallel manipulator has been created (Fig.2), which shows the lower 1 and upper 2 platform, united by six hydraulic actuators 3, the control valve 4 , a pump station 5. In this case, to change the lengths of the connecting links, unilateral controlled hydraulic actuators are used.

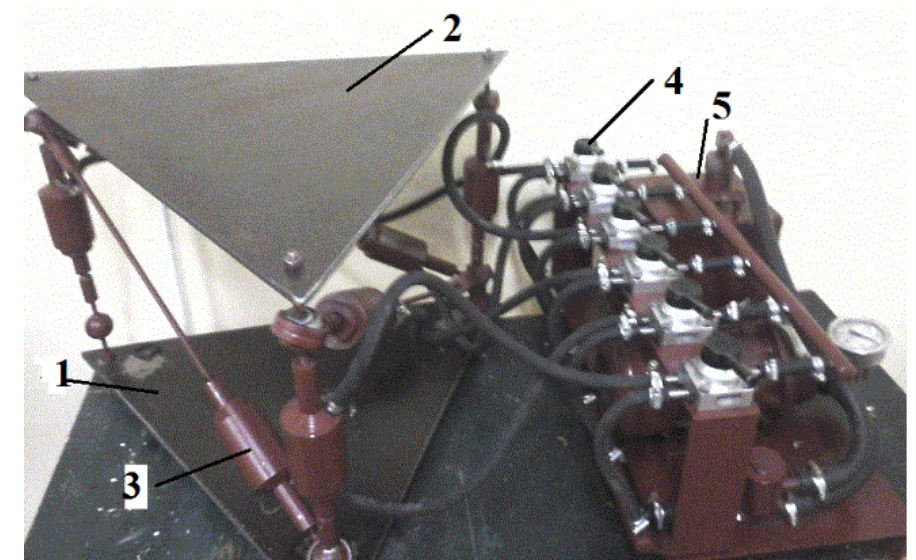

Fig. 2Working model of the parallel manipulator

The working model of the parallel manipulator with platforms 1 and 2 has demonstrated its unique properties and functionalities due to the peculiarities of the topological structure. First, each of the six drives can move independently of the others. For example, it is possible to change length of one CL using the actuator without changing the lengths of the other CL, whereas the upper platform takes a strictly predefined position with regards to the lower platform.

Second, by changing the lengths of a certain set of CL in a prespecified way, it is possible to obtain the required spatial position of the movable platform or its simplest movements with regards to the lower platform. For example, the movement of platform 2 relative to platform 1 can be achieved by positioning some direct $\mathrm{B} 2 \mathrm{C} 2$ (Fig.1). At the beginning, unit $\mathrm{C} 2$ moves to the given spatial point through the change in the lengths of CL 3,6, and 7; next, unit B2 moves because of a change in the lengths of CL 5 and 8. The positioning of the upper platform against the lower one is achieved by turning unit A2 around in-line with B2C2 due to the change in length of CL 4. Therefore, the spatial positioning of platform 2 with regards to platform 1 can be divided into three successive phases: movement of unit $\mathrm{C} 2$, movement of unit B2 and movement of unit A2. If the lengths of connecting links 3,6, and 7 are not changed, but the lengths of the other connecting links are changed, then the upper platform 2 will make spherical rotations around unit C2. By changing only the length of link 4, it is possible to cause rotational motion of platform 2 with regard to the axis going through units $\mathrm{C} 2$ and B2. To obtain the translational motion of platform 2, you must change the length of each CL. Thus, the resulting design of the parallel manipulator enables targeted control of the motion of the upper platform. Third, the structure topology of the new platform manipulator ensures solving of the direct and inverse kinematic problems. Moreover, the solution of the inverse problem does not appear to be complex. The solution of the direct kinematic problem, which is not solved explicitly for other platform manipulators, was obtained by the author in previous works [7,8]. None of the known platform type robots with degrees of freedom equal to six have the above properties. Due to these properties and capabilities, this platform type robot can be used as an active controlled support, installed between the protected object and the source of sudden mechanical impact.

\section{The platform type robot as active support}

A sudden mechanical impact is as a force or kinematic impact, which results in unwanted effects.We can include large-scale earth pulsations in different planes during earthquakes, 
fluctuations of water surface affecting floating crafts, and strokes during landing of the aircrafts in sudden mechanical impacts. In this paper, new robot functionality is examined for the purpose of scientific substantiation of the use of the platform type robots as active supports with six axes. To be used as an active support and to ensure high capacity, the platform type robot is applied to automatically controlled hydraulic drives (controllable shock absorbers).

The mechanism to damp the sudden mechanical impact is based on the fact that it is possible at any time to achieve the balance of forces applied to the upper platform by controlling the pressures in the cavities of hydraulic cylinders, i.e., immovability of the upper platform, when the lower platform makes unwanted movements. The required pressures in the above piston cavities of the hydraulic cylinders are maintained using delivery (normally open) and bleeder (normally closed) controlled solenoid valves. Fig. 3 shows the schematic of the automatically controlled hydraulic drive of the platform type robot installed between the protected facility 1 and source 2 . The drive of the active support consists of basement 3, upper platform 4 and side-driving hydraulic cylinder 5 , with piston 6 and $\operatorname{rod} 7$.

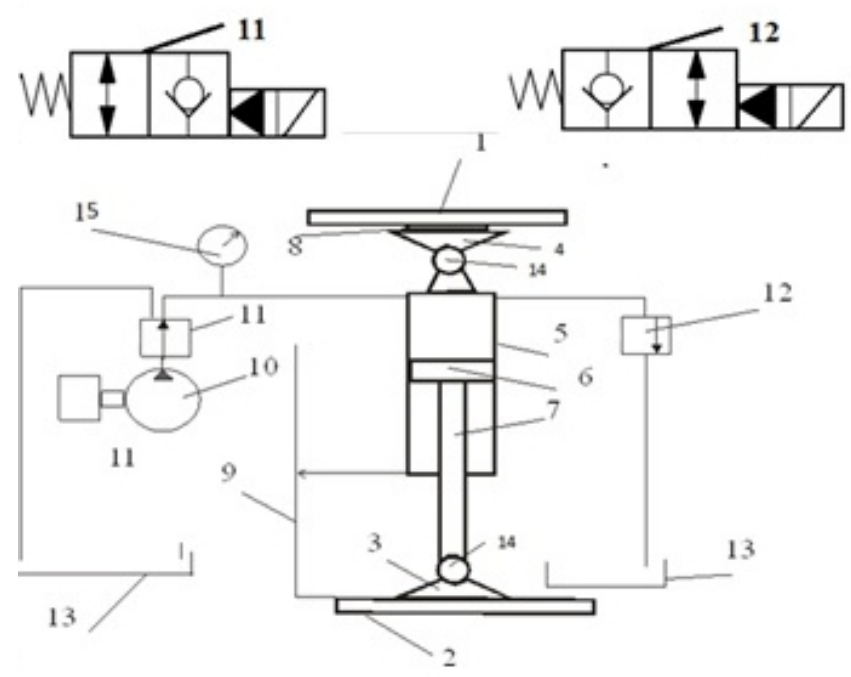

Fig. 3 Schematic of the electro-hydraulic drive

Between the upper platform 4 and the object is mounted strain gauge sensor 8 , which measures the force acting on the platform. The rod's movement is measured using movement sensor 9. The hydraulic system consists of pump station 10, normally open delivery solenoid valve 11, normally closed bleeder solenoid valve 12, and tank 13. Pressure in the cylinder cavity is measured using sensor 15; the upper and lower platforms are connected with a hydraulic cylinder with spherical joints 14. The work of the hydraulic driver is based on automatically providing the necessary pressure on the piston area of the hydraulic cylinder. Depending on the external load, defined by strain gauge 8 , and the position of the upper platform, defined by the displacement sensor 9, the algorithm can calculate the required pressure of the fluid in the over piston area, which is compared with the actual pressure determined by sensor 15 . The signal for triggering an automatic system to maintain the required pressure is the discrepancy between the actual pressure and the pressure measured by sensor 15. If there are discrepancies, then through delivery valve 11 and bleeder valve 12 , the required pressure above the piston cavity of hydraulic cylinder is automatically set. Fig. 4 shows the experimental assembly of the platform type robot, which is fitted with pressure sensors 3, magnetostrictive movement sensors 2 , and straingage sensor 1 . 


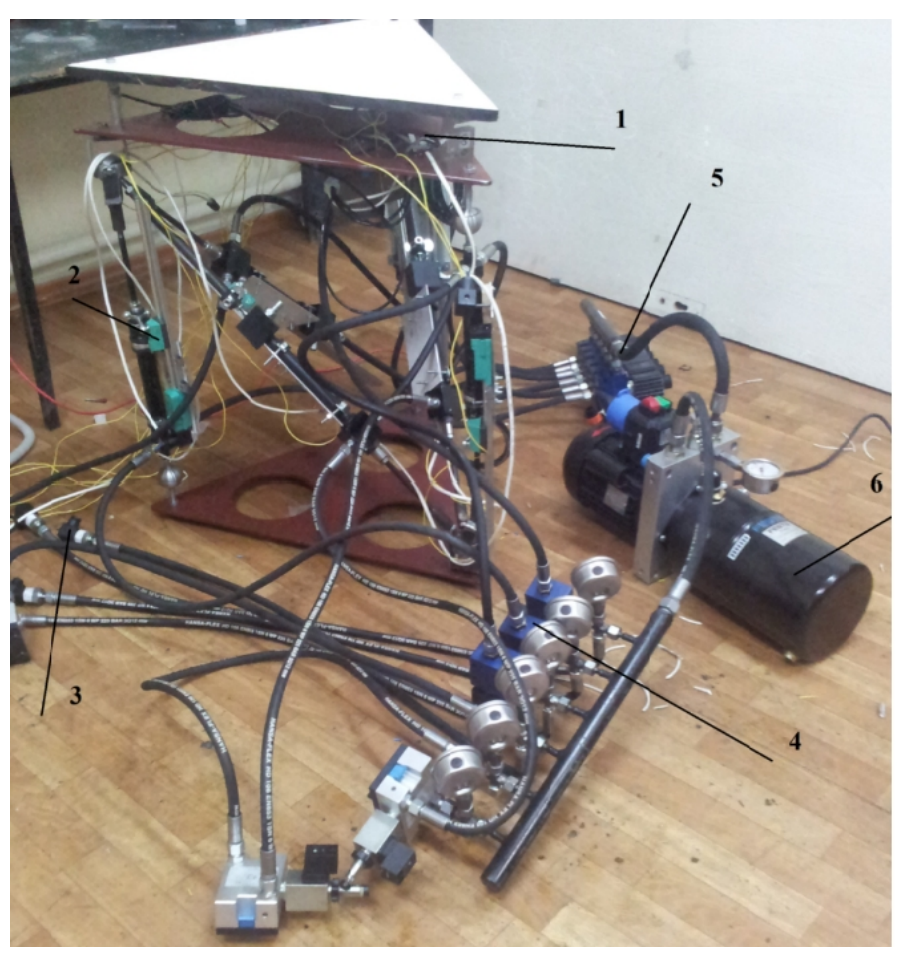

Fig. 4 Experimental assembly of the platform type robot

Delivery valve 4 and bleeder solenoid valves 5 act as the executors of this system. The hydraulic system is fed by hydraulic pump 6. To automate the robot's operation as an active support, a control system (CS) has been designed. Its functional block scheme is shown in Fig. 5.

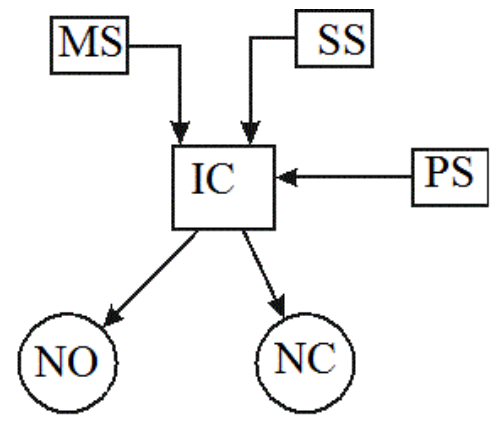

Fig. 5 Function block scheme of the control system

The following symbolism is used in Fig. 5: IC - industrial controller; MS - movement sensor; SS - strain-gage sensor; PS - pressure sensor; NO - normally opened delivery valve; NC - normally closed bleeder valve. The control system CS acts as follows: the signal from the movement sensor and from the strain-gage sensor is received by the industrial controller on a real time basis. CS acts as follows: the signal from the SS and from the PS in real time goes to the IC. Based on an existing algorithm, IC determines the necessary pressure PN in the cavities of the cylinders to balance the working power. IC compares that pressure with the actual pressure of above the piston cavity of the hydraulic cylinder PA, according to the data from the detector PS, and as a result, generates a control signal that is fed to one of the valves. Thus, if PA $>$ PN, then the bleeder valve NC is opened, and the delivery is closed; if on the contrary, the delivery valve is opened and the bleeder valve is closed. In the case where the pressure is $\mathrm{PA}=\mathrm{RN}$, then the condition of the valves does not change. In the case when the rod is in its extreme uppermost position or the lowermost position, the valve position can be changed in only a certain way or does not change at all. In IC, there is a program to prevent the possible collision of the piston with the case when reaching the end position. The source for this 
program is the signals from the movement sensors MS. The current parameters of the system and the valves' operating modes are displayed by the SCADA imaging system on the PC.

\section{Research on the motor reaction}

One of the topical objectives of modern robot technics is to create robots with capabilities exceeding those of humans, which will be able to perform the given functions in extreme conditions or when human capabilities are limited. For example, human capabilities related to the motor reaction (human response time) exceed $200 \mathrm{~ms}$ [9]. For some robots, the required motor reaction is the main functional objective. For example, if a platform type robot is used as an active support, it responds rapidly to the source of sudden impacts and takes measures to prevent such impact on the protected item. In this case, the functionality of the adaptive support substantially depends on its motor reaction.

Motor reaction (MR) is considered to [10] be the shortest time of response with a simple and known in advance movement to a sudden but known signal. We assume that the motor reaction of the platform type robot depends on the motor reaction of the controllable hydraulic drives. Therefore, the issue related to the determination of the motor reaction of a separate controllable drive is first considered below. The controllable hydraulic drive includes elements of a different physical nature and functions to be fulfilled. For example: computer software and hardware for automated control; solenoid valve; the line, which moves the working fluid (WF); executive device in the form of a unilateral action hydraulic cylinder with a rod and return spring. The said elements in aggregate affect the motor reaction of the hydraulic drive. At the same time, due to the high-speed response of the modern microprocessor machinery and smallness of the motor reaction control hardware and software, we pay special attention to the research of two elements, namely the solenoid valve and the power element of the hydraulic cylinder. It is assumed here that the motor reaction of these elements depends on the time constant, in the case where each of these elements is considered as a dynamic link of the control system. In this case, the motor reaction of the controlled hydraulic drive is equal to a certain portion of the sum of two constant links - the solenoid valve and the executive device. Therefore, the analysis of the dynamic elements is given below, and constant time is determined for each link through a computer generated simulation.

Solenoid valve as a dynamic link. The solenoid valve consists of electromagnetic and mechanical component parts [11]. For the electromagnetic component, it is possible to use the following linearized differential equation of an operator form:

$$
\mathrm{RI}+\mathrm{LpI}=\mathrm{U}-\mathrm{k}_{\mathrm{E}} \mathrm{px}
$$

where R- aggregate active resistance of the control winding and source control; U- input voltage of the valve; L- inductance control of the winding; $k_{E^{-}}$back emf ratio; x- movement of the electromagnet armature; and I - control current.

The tractive effort $P_{M}$ of the electromagnet is calculated using the following equation:

$$
\mathrm{P}_{\mathrm{M}}=\mathrm{k}_{\mathrm{M}} \mathrm{I},
$$

wherek $_{\mathrm{M}^{-}}$gear ratio.

For the mechanical part of the solenoid valve, it is possible to use the following differential equation of the second order, in the operator form:

$$
\mathrm{Mp}^{2} \mathrm{x}+\mu \mathrm{px}+\mathrm{cx}=\mathrm{P}_{\mathrm{M}}+\mathrm{P}_{\mathrm{R}},(3)
$$


where M-the anchor mass and cited mass of the moving elements of the mechanical component of the valve; $\mu$ - viscosity of the WF; $\mathrm{c}$-aggregate rigidness of the mechanical and magnetic spring; and $\mathrm{P}_{\mathrm{R}}$ - the anchor force from the action of forces applied to the mechanical parts of the valve.

By solving equations (Eq. 1- Eq. 3) together, we obtain the equation for the solenoid valve as a dynamic link as follows:

$$
\mathrm{A}(\mathrm{p}) \mathrm{x}=\mathrm{k}_{1} \mathrm{U}+\mathrm{k}_{2} \mathrm{~B}(\mathrm{p})+\mathrm{P},
$$

where $\mathrm{A}(\mathrm{p})$ - own system operator; $\mathrm{k}_{1}, \mathrm{k}_{2}$-transmission coefficients; and $\mathrm{B}(\mathrm{p})$-input system operator.

The variables included in the summarized equation of the solenoid valve as a dynamic link are determined by the following functional connections:

$$
\begin{aligned}
& A(p)=\frac{L M}{R c} p^{3}+\frac{\mu L+M R}{R c} p^{2}+ \\
& +\left(\frac{L}{R}+\frac{\mu}{c}+\frac{k_{E} k_{M}}{R c}\right) p+1 ; \\
& B(p)=\frac{L / R}{c} ; k_{1}=\frac{k_{M}}{R c} ; k_{2}=1 / c .
\end{aligned}
$$

According to equation (Eq. 4), the structure scheme of the solenoid valve as a dynamic link is created. In the result of the model based analysis, a transient curve is created in Simulink Matlab, and constant time $T_{1}$ is determined. Computer modelling can be used for the design of the drive or, to compare, when the constant physical and mechanical parameters included in expressions (Eq. 3) and (Eq. 4) are known.

Executive device of the hydraulic driver - as a dynamic link. The main element of the controllable hydraulic drive is the executive device (Fig. 6) as a system consisting of cylinder 1, rod 2, and piston 3 , with orifice holes 4 , lower cavity of the cylinder 5 , above piston cavity of the cylinder 6 with the WF, and the return spring 7 .

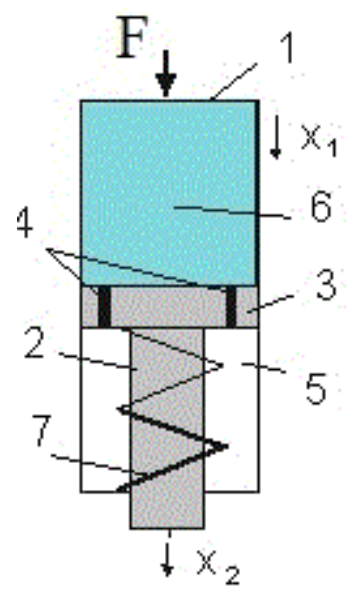

Fig. 6 Executive device of the hydraulic drive with the piston channel

To simplify the non-linear and complicated dynamics equation, we disregard the frictional force. Then, based on conditions of the piston's kinaesthetic balance, we have the following functional connection in the operator form:

$$
\begin{aligned}
& \operatorname{Mp}^{2}\left(\mathrm{x}_{1}-\mathrm{x}_{2}\right)-\mu \mathrm{p}\left(\mathrm{x}_{1}-\mathrm{x}_{2}\right)- \\
& \mathrm{c}\left(\mathrm{x}_{1}-\mathrm{x}_{2}\right)=\mathrm{F}-\mathrm{PS},
\end{aligned}
$$


whereM- mass of moving parts of the mechanical component cited to the rod with the piston; $\mu$ viscosity of the WF; $c$-spring power, F-load by external forces cited to the rod; and P-pressure in thedrive hydro system. Taking a laminar mode of motion WF, we define the rate of fluid flowing through the throttling holes as the result of the pressure without taking into account the compressibility WF:

$$
\mathrm{Q}=\mathrm{kP}+\mathrm{Sp}\left(\mathrm{x}_{1}-\mathrm{x}_{2}\right)
$$

By solving equations (Eq. 5 and Eq. 6) altogether, we obtain the following equation of the dynamic link in the operator form:

$$
\begin{aligned}
& (\mathrm{Mp}+\mathrm{f})(\mathrm{vp}+\tau)+1) \mathrm{u}=\mathrm{k}_{1}\left(\mathrm{x}_{1}-\mathrm{x}_{2}\right)- \\
& -(\mathrm{vp}+\tau)(\mathrm{F}-\mathrm{PS})]
\end{aligned}
$$

wheref-the ratio of the power; $\mathrm{v}=\mathrm{V} / 2 \mathrm{ES}^{2}$ - spring power; $\mathrm{V}, \mathrm{E}$ - volume above the piston and elasticity of the WF; and $\mathrm{k}_{1}, \tau$-transmissioncoefficients. Eq. 7 is the structure scheme of the dynamic link, and as a result of the computer generated simulation using Matlab Simulink and the transient curve, the time constant $\mathrm{T}_{2}$ is established for the executive device of the hydraulic drive. As indicated above, computer modelling can be used to design drivers or compare them when the constant values in equations (Eq. 5- Eq. 7) are known.

Motor reaction requires time, which is significantly less than the constant time because it characterizes the time at which the output value reaches the minimum changes in the state. Therefore, in a first approximation, it is possible to think that motor reaction is equal to $1 / 3$ of the constant time. Thus, the motor reaction of the hydraulic drive is generally equal to $\mathrm{D}=1 / 3\left(\mathrm{~T}_{1}+\mathrm{T}_{2}\right)$. This indicator may be used as a comparison characteristic when choosing and designing the drives.For the analysis of motor reaction, experiments of real drives and experimental study of the dependence on time of the simultaneous change of pressure WF in the pressure line and as a result of displacement of the rods of the hydraulic drive were conducted.Robot SHOLKOR was used as the experimental assembly (Fig.4). To perform the experiments, a load of a given weight $(15 \mathrm{~kg})$ was placed on the upper platform of the robot. Movement sensors were installed in the middle position of the rods when they were put forward at half stroke. Using the strain gauges and pressure sensors, the values acting on the force and the pressures in the working cavities of the hydraulic cylinders were determined. These values were processed by the controller and used by the software to control the robot. Thereafter, according to the given sequences, the control module controlled the movement of the robot's platform. Data received from theinformation-measuring system were processed by thecontrolleraccording to the set program, and graphs of the displacement of the rod and the pressure in the hydraulic cylinder were displayed on the monitor screen in the SCADA system. Some of the obtained experimental data are given inFig. $7(a, b)$. It shows the curves of the experimental studies obtained for the two drives of the platform type robot SHOLKOR, in particular, for the hydraulic drive 6- $a$ );7-b). The graphs obtained in the SCADA system show some of the dependencies of the displacement (red line) and pressure (blue line) on time over a period of time equal to $2 \mathrm{~min}$. In hydraulic drive 6 , the diameter of the piston of the hydraulic cylinder, $d_{1}=16 \mathrm{~mm}$, whereas the diameter of the piston in hydraulic drive 7 is $d_{2}=20 \mathrm{~mm}$. Both hydraulic drives are single-sided and have a pull-back spring. In hydraulic drive 7, the piston has a throttled channel; in hydraulic drive 6 , there is no channel.To more accuratelydisplay the time on the graphs obtained in real time, the axis is divided into 620 equidistant segments. Thus, the scale has a division value of 0.19 s. Ultimately, the curves shown in Fig.7, a proves that the rod begins to move $0.76 \mathrm{~s}$ after the power fluid pressure is generated in the cavity of the hydraulic cylinder.During experiments with hydraulic drive 6, the state of the delivery valve was observed. Additionally, Fig. 7, $b$ shows the time of opening of the pressure valve, which corresponds to the end of the solid red line. 


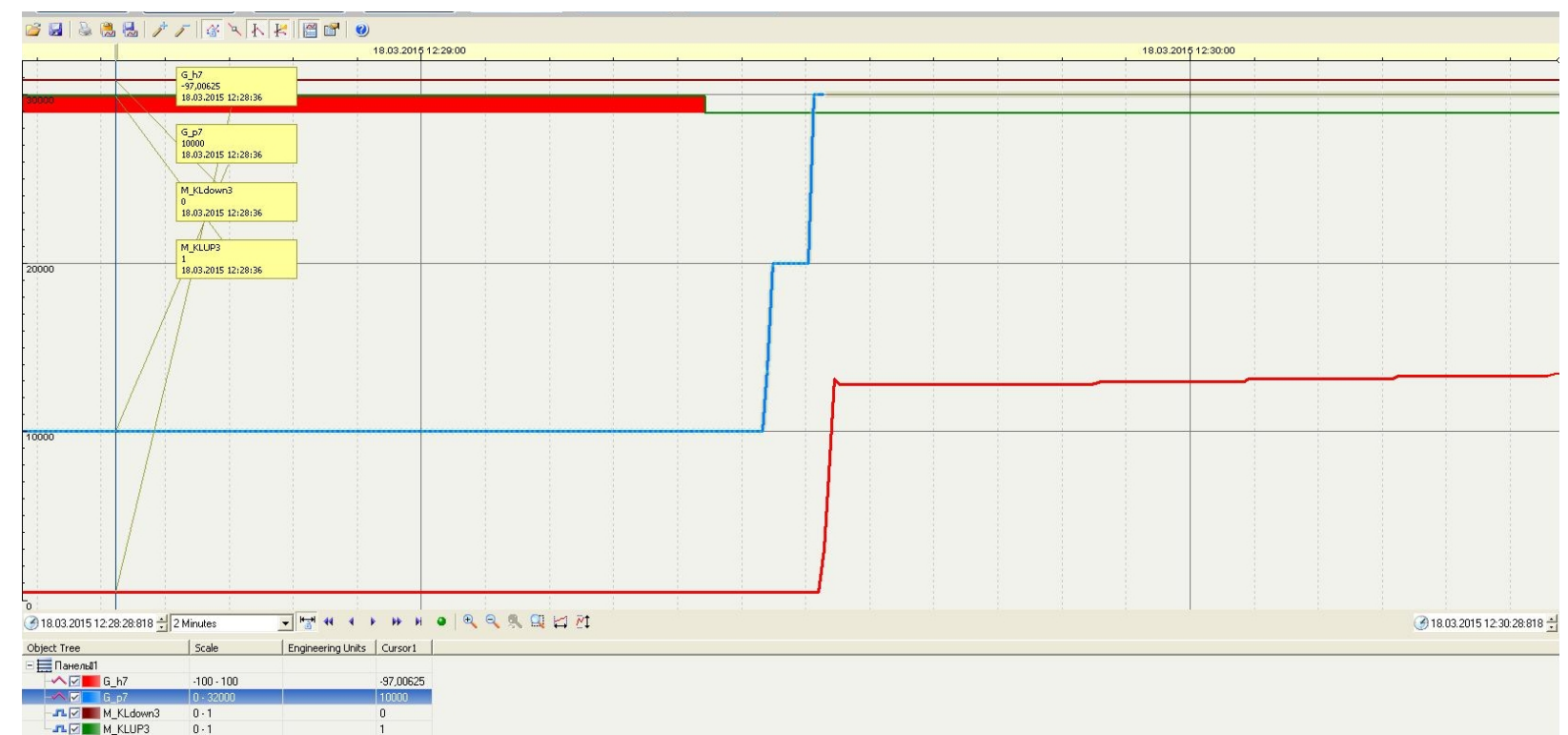

a)

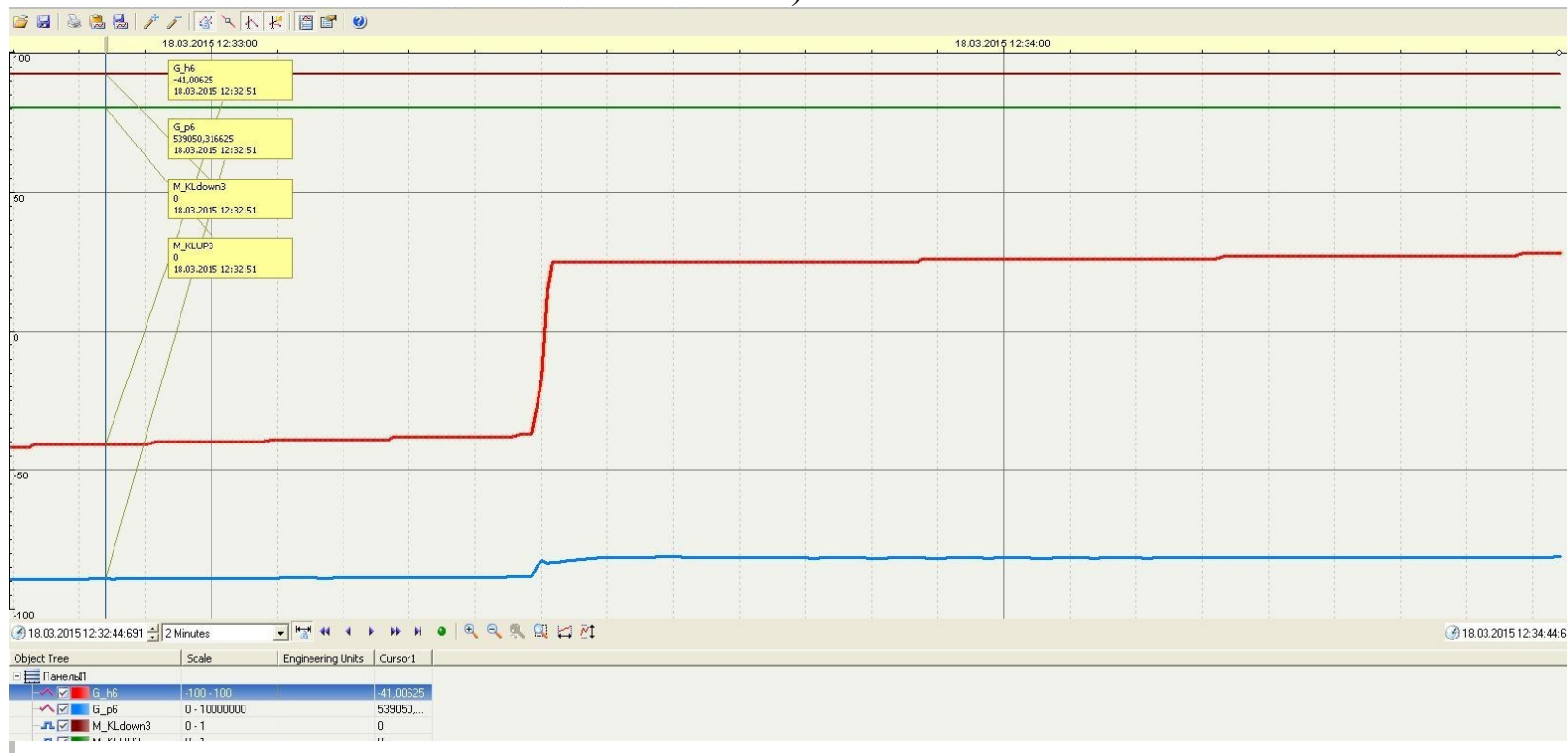

b)

Fig. 7 Experimental studies' curves

From the graphs, it follows that in $4.5 \mathrm{~s}$ after opening of the valve, a pressure change begins in the cavity of the hydraulic cylinders. This lag is explained by the resistance of the main line WF. By comparing the graphs of the pressure and displacement, it can be observed that the movement of the rod begins $4.56 \mathrm{~s}$ after receiving the pressure in the cylinder cavity. The obtained time data describing the motor reactions of various dynamiclinks as the components of the hydraulic drives allow for the following conclusions: the main line WF has the largest motor reaction; the motor reaction of the hydraulic drive with throttled channels in the piston is 6 times larger than the drive without the channel. This means that to improve the motor reaction of the drive, it is required to ensure joint operation of the executors in the hydraulic drive and control equipment, i.e., it is required to use mechatronic hydraulic drives to eliminate the impact of the main line WF. On the other hand, experiments have shown that it is undesirable to use throttling elements in the hydraulic drive of a platform type robot used as active support. This lag is explained by the resistance of the main line WF. By comparing the graphs of the pressure and displacement, it can be observed that the movement of the rod begins $4.56 \mathrm{~s}$ after receiving the pressure in the cylinder cavity. The obtained time data describing the motor reactions of various dynamic links as the components of the hydraulic drives allow for the following conclusions: the main line WF has the largest motor reaction; the motor 
reaction of the hydraulic drive with throttled channels in the piston is 6 times larger than the drive without the channel. This means that to improve the motor reaction of the drive, it is required to ensure joint operation of the executors in the hydraulic drive and control equipment, i.e., it is required to use mechatronic hydraulic drives to eliminate the impact of the main line WF. On the other hand, experiments have shown that it is undesirable to use throttling elements in the hydraulic drive of a platform type robot used as active support. The experimental studies' findings showed that it is possible to achieve the required values of motor reactions for a platform type robot to be used as an active support.

\section{Conclusion}

Based on the new structure topology and application of the multilink spherical joint, the current model of a new platform type robot with six degrees of freedom has been creased. Based on the working model, as well as the solution of the direct kinematic problem, it has been proven that the new robot has a number of material advantages versus the Gough-Stewart platforms. First, each of the six drives can move independently of the others. Second, by changing the lengths with actuators of a certain set of connecting links in a specified way, it is possible to obtain any desired spatial position of the movable platform with regards to the stationary platform. Third, the structure topology of the new platform type manipulator ensures solution of direct kinematic problems. Due to the said properties and capabilities, this platform type robot can be used as an active controllable support to be installed between the protected item and the source of sudden mechanical impacts. However, for the platform type robot to be used as an active support, the said capabilities are insufficient. In addition, the platform type robot must be able to respond on a timely basis to exposure to the source of sudden impacts to prevent any effect on the protected object. This functionality of the platform type robot is substantially predetermined by its motor reaction, which depends on the controllable drives' motor reactions. The article refers to the possibility to study the motor reaction when designing and choosing the drives by considering the automatically controlled hydraulic drive to be a system consisting of an assembly of two dynamic links. Thus, on the basis of the dynamic equations of the links, the structural schemes were composed. Using computer generated simulation of the links' dynamic characteristics, the constant time can be determined to find the motor reaction of the system, which can be used as a comparative characteristic when selecting and designing drivers.

The real value of motor responses was investigated for two types of single-side hydraulic drivers: with a throttling channel in the piston and without a channel. Furthermore, experimental research has been conducted using the platform type robot as an experimental assembly. The obtained experimental values of the motor reactions of the different hydraulic drives allow us to make the following conclusions: the main line WF has the largest motor reaction; the motor reaction of the hydraulic drive with channels in its piston is 6 times larger than that of the hydraulic drive without channels. This suggests that to improve the drive's motor reaction, mechatronic hydraulic drives without throttles are required because the application of the throttled elements in the hydraulic drives considerably (6 times) increases the motor reaction of the drive. The results of the computer-based research show that it is possible to determine the motor reactions of the platform type robot and to achieve the required values of the motor reactions during the design phase to ensure that the platform type robot can be used as an active support.

The experimental studies' findings provide recommendations pertaining to the drives of the platform type robot when used as an active support.

\section{References}

[1] V.E. Gough, Contribution to discussion of papers on research in Automobile Stability, Control and Tyre Performance. Proc. Auto Div. Inst. Mech. Eng. (1956) 392-394.

[2] D. A.,Stewart, Platform with six degrees of freedom, Proceedings of the Institution of Mechanical Engineers, London, Vol. 18,15 (1965) 371-385. 
[3] B. Dasgupta, T.S. Mruthyunjaya, The Stewart platform manipulator: a review, J. Mechanism and Machine Theory 35, (2000) 15-40.

[4] P. Nanua, K.J. Waldron, V. Murthy, Direct Kinematic Solution of a Stewart platform, IEEE Trans. On Robotics and Automation, Vol. 6 (1990) 438-444.

[5] J. Angeles, Fundamentals of Robotic Mechanical Systems: Theory, Methods and Algorithms, second ed., Springer, 2002.

[6] J. P. Merlet, Parallel Robots, Springer, Dordrecht, 2006.

[7] K.S. Sholanov, Manipulator of a Platform Type Robot Sholkor, J. Advanced Materials Research, Vol. 930 (2014) 321-326.

[8] K.S. Sholanov, Synthesis of the Schematic Structure and Solving the Task of Positioning of a Parallel Platform Manipulator, J. Mechatronics, Automation and Control, 11, Moscow (2014) 44-50.

[9] H.T. Schie, et al., Evidence for fast, low-level motor resonance to action observation, MEG study. Social Neuroscience, 3 (2008) 213-28.

[10] G.G. Malinetsky, Robotechnics, Prediction, Programming, LKI Publishing House, Moscow, 2008.

[11] V.F. Kazmirenko, Electro-Hydraulic Mechatronic Motion Modules. Foundations of the Theory and System Design, Radio and Svyaz, Moscow, 2001. 